\title{
Hepatitis C Crónica y usuarios con un historial de inyección de drogas en España: evaluación de la población, retos para un tratamiento efectivo
}

\section{Chronic Hepatitis C and people with a history of injecting drugs in Spain: population assessment, challenges for effective treatment}

\author{
Roncero, Carlos*; Vega, Pablo**; Martinez-Raga, Jose***; Torrens, Marta****. \\ * Addiction and Dual Diagnosis Unit, Department of Psychiatry, Vall d'Hebron University Hospital - Public Health Agency, \\ Barcelona (ASPB), CIBERSAM. Barcelona, Spain. Department of Psychiatry and Legal Medicine, Universidad Autonoma de \\ Barcelona, Barcelona, Spain; ** Sociedad Española de Patología Dual, Spain. Instituto de Adicciones, Madrid, Spain \\ *** Teaching Unit of Psychiatry and Psychological Medicine, University Hospital Doctor Peset \& University of Valencia, \\ Valencia, Spain. University Cardenal Herrera CEU, Valencia, Spain; **** Institut de Neuropsiquiatria i Addiccions, Hospital \\ del Mar, Barcelona, Spain. IMIM- Hospital del Mar Medical Research Institut, Barcelona, Spain. Department of Psychiatry and \\ Legal Medicine, Universidad Autonoma de Barcelona, Barcelona, Spain.
}

L a hepatitis C crónica (HCC) es una enfermedad de desigualdad que afecta desproporcionadamente a las personas desatendidas por la salud pública. Personas con antecedentes de uso de opioides o uso de drogas por vía parenteral (UDVP) se enfrentan a desigualdad y estigma. Una historia previa de inyección de drogas es un comportamiento que incrementa el riesgo de contraer el Virus de la Hepatitis C (VHC). Hay una gran incidencia de VHC entre las personas se inyectan drogas. Hay más de 300,000 personas en España que actualmente se inyectan drogas o tienen antecedentes (Ministerio de Sanidad Servicios Sociales e igualdad, 2014). Hay aproximadamente 100,000 personas en España con un historial trastorno de uso de opioides y la mayoría son UDVP o ex UDVP (EMCDDA, 2016). Aproximadamente 80,000 personas usan los servicios para tratar el trastorno de uso de opioides cada año (Torrens, Fonseca, Castillo, y Domingo-Salvany, 2013).

La presencia de VHC en esta población es de 50-80\% (Muga et al., 2015). Un 32\% de las personas continúan inyectándose drogas durante el tratamiento en España (Roncero et al., 2011). Sin embargo, los usuarios de drogas en tratamiento presentan un menor riego de infección por el VHC, debido a que son menos propensos a compartir agujas que aquellos fuera del tratamiento (Donmall, Jones, Davies, y Barnard, 2009).
En España, se han tratado un número muy limitado de UDVP con VHC (Muga et al., 2015). La tolerabilidad y efectividad de la terapia del VHC en el pasado ha limitado las intervenciones exitosas. Sin embargo actualmente hay nuevos tratamientos eficientes y accesibles (Grebely et al., 2015). En general, el tratamiento de los UDVP es menos probable que para otros pacientes (Mravčík et al., 2013). Muchos de los UDVP asumen que no pueden acceder al tratamiento para el VHC por sus problemas de salud y las desigualdades del sistema; probablemente hay una inutilidad percibida en solicitar pruebas de detección y participar en el tratamiento. Además, en el pasado la ausencia de nuevos tratamientos y el miedo a los efectos secundarios de las antiguas medicaciones usadas para el tratamiento de VHC, como el interferón, limitaba el acceso (McGowan y Fried, 2012). Por otra parte, el escaso conocimiento sobre la infección del VHC y sus secuelas son limitaciones que se deben considerar. Estos problemas deben de ser solucionados con nuevas y diferentes formas de educación de la salud. Por ejemplo, el uso de grupos de apoyo conducidos por pares son probablemente de gran importancia. También se puede considerar la posibilidad de facilitar el diagnóstico por pares o el autodiagnóstico en ciertos establecimientos, incluyendo sitios informales y no clínicos como farmacias, programas de intercambios de jeringuillas y centros de servicios sociales (Rose, Lutnick, y Kral, 2014).

Recibido: Noviembre 2016; Aceptado: Diciembre 2016.

Enviar correspondencia a:

Roncero, C. Addiction and Dual Diagnosis Unit. Department of Psychiatry, Vall d'Hebron University Hospital, Passeig Vall d'Hebron, 119-129, 08035 Barcelona, Spain. E-mail: croncero@vhebron.net. 


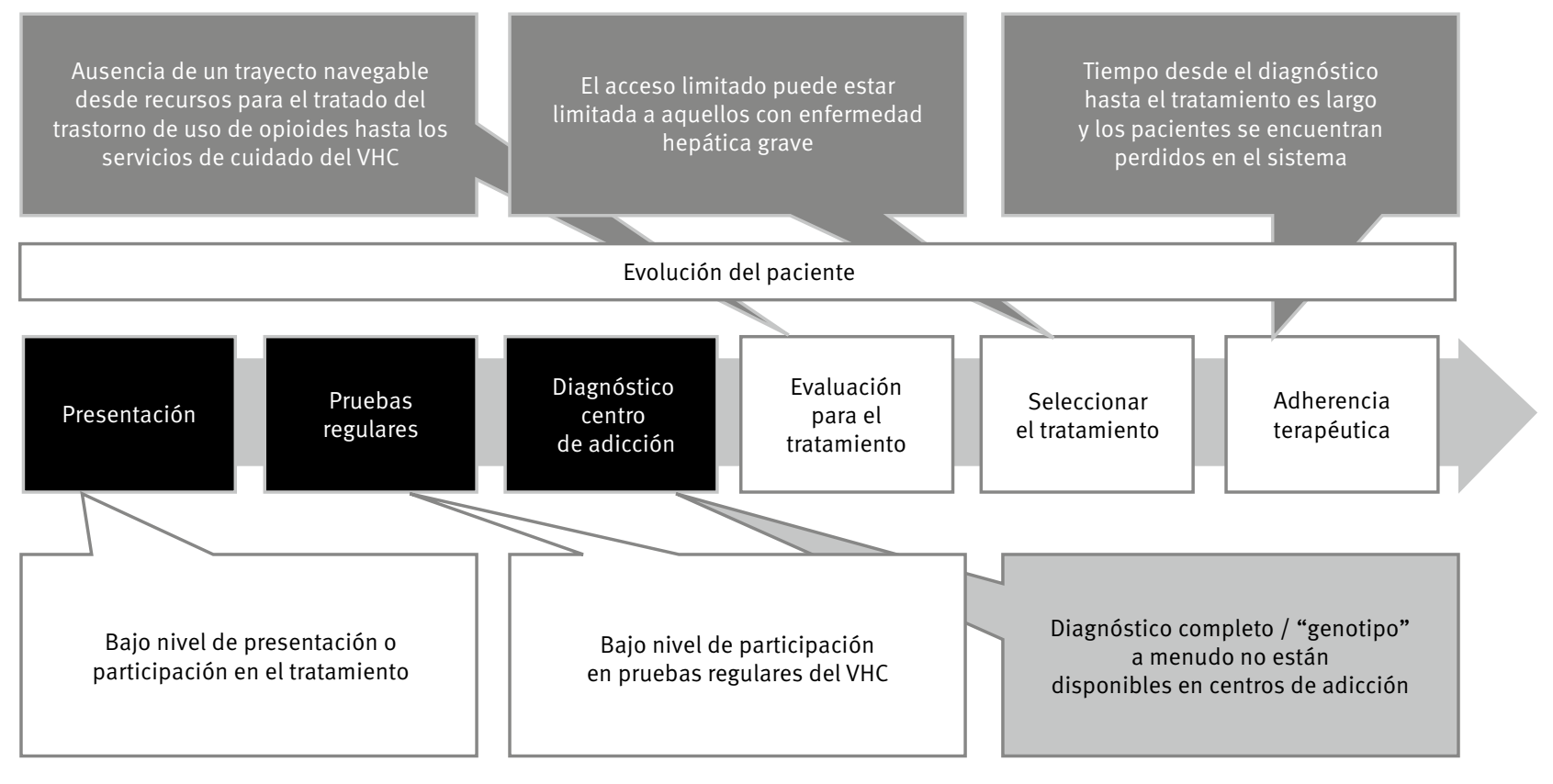

Factores limitantes más importantes

Fig. 1. Evolución del paciente

La barrera más importante para el acceso al tratamiento del VHC para esta población es el enlace entre los centros de tratamiento de las drogodependencias y los centros que tratan el VHC (unidades o servicios de enfermedades infecciosas y/o de hepatología). La separación de estos servicios, que están definidos por la organización e infraestructura del sistema sanitario de España, es la mayor limitación para la administración con éxito del tratamiento de los pacientes con VHC. Estas limitaciones pueden ser solucionadas facilitando una colaboración entre las unidades que tratan a los pacientes adictos y los servicios de enfermedades infecciosas y/o hepatología, incluyendo el desarrollo de un protocolo conjunto para los profesionales sanitarios y los pacientes.

Es muy difícil entender cómo se puede tratar efectivamente a estos grupos ya que muchos de ellos no se relacionan con servicios sociales y sanitarios en absoluto, o solo con servicios para el tratamiento de la drogodependencia a los opioides. Se han descrito las siguientes causas que explicarían el acceso limitado al tratamiento del paciente: (1) bajo nivel de presentación de los síntomas y participación en el tratamiento, (2) baja participación en pruebas regulares del VHC, (3) clara ausencia de un protocolo claro desde los recursos para el tratamiento del trastorno de uso de opiáceos hasta los servicios de tratamiento del VHC para muchos pacientes (Figura 1).

\section{Conclusiones}

Hay aproximadamente 150,000 personas en España con trastorno de uso de opiáceo y muchos de ellos también tienen un historial de inyección de drogas. De esta población, hay 80,000 personas que se relacionan con los servicios para el tratamiento de la drogodependencia, recibiendo tratamiento sustitutivo con opiáceos y otros tipos de intervenciones. Sin embargo, hasta la actualidad, pocos UDVP con VHC en España han sido tratados. Las razones por las que el numero de UDVP tratados es bajo apunta a una serie de estrategias que pueden mejorar el acceso a servicios sanitarios de estos grupos. Hay una oportunidad para los responsables políticos, médicos y pacientes para hacer un gran cambio en la manera que se trata el VHC en UDVP.

\section{Reconocimientos}

Se quiere agradecer a los 21 expertos de toda España que contribuyeron a este trabajo. Se recibió asistencia editorial por Tara Lumley de la empresa applied strategic, London (UK). Se recibió una beca de Gilead, que no tuvo ninguna influencia en el desarrollo del trabajo o la redacción del manuscrito.

\section{Conflicto de intereses}

El Dr Carlos Roncero ha sido ponente para Janssen-Cilag, Ferrer-Brainfarma, Pfizer, Reckitt-Benckiser/Indivior, Lundbeck, Otsuka, Servier, Shire, Lilly, GSK, y Astra. Ha participado como miembro de boards de Janssen- Cilag, Indivior y Gilead. Desarrolló el proyecto PROTEUS con una beca de Reckitt-Benckisert/INDIVIOR. No hay otros conflictos de interés que declarar que puedan afectar a este artículo. El Dr Pablo Vega ha sido ponente para JanssenCilag, Servier, Lundbeck, Indivior, Lilly and Gilead. No hay 
otros conflictos de interés que declarar que puedan afectar a este artículo. El Dr Martínez-Raga ha sido ponente para Janssen- Cilag, Servier, Lundbeck, y Lilly. No hay otros conflictos de interés que declarar que puedan afectar a este artículo. La Dra Marta Torrens ha recibido honorarios por consultorías con Lundbeck, Mundipharma, Indivior y Gilead. No hay otros conflictos de interés que declarar que puedan afectar a este artículo.

\section{Referencias}

Donmall, M., Jones, A., Davies, L. y Barnard, M. (2009). Summary of key findings from the Drug Treatment Outcomes Research Study (DTORS).

EMCDDA. (2016). European Drug Report: Trends and Developments 2016. Luxembourg.

Grebely, J., Robaeys, G., Bruggmann, P., Aghemo, A., Backmund, M., Bruneau, J., ... Dore, G. J. (2015). Recommendations for the management of hepatitis $\mathrm{C}$ virus infection among people who inject drugs. The International Journal on Drug Policy, 26, 1028-1038. doi:10.1016/j. drugpo.2015.07.005.

McGowan, C. E. y Fried, M. W. (2012). Barriers to hepatitis C treatment. Liver International : Official Journal of the International Association for the Study of the Liver, 32 Suppl 1, 151-156. doi:10.1111/j.1478-3231.2011.02706.x.

Ministerio de Sanidad Servicios Sociales e igualdad. (2014). 2014 National Report (2013 data) to the EMCDDA. Spain New Development, Trends.

Mravčík, V., Strada, L., Štolfa, J., Bencko, V., Groshkova, T., Reimer, J. y Schulte, B. (2013). Factors associated with uptake, adherence, and efficacy of hepatitis C treatment in people who inject drugs: A literature review. Patient Preference and Adherence, 7, 1067-1075. doi:10.2147/PPA. S49113.

Muga, R., Zuluaga, P., Sanvisens, A., Rivas, I., Fuster, D., Bolao, F. y Tor, J. (2015). Hepatitis C associated to substance abuse: ever closer to a treatment without Interferon. Adicciones, 27, 141-149.

Roncero, C., Fuste, G., Barral, C., Rodríguez-cintas, L., Martínez-Luna, N., José, E.O. F. y Casas, M. (2011). Therapeutic management and comorbidities in opiate-dependent patients undergoing a replacement therapy programme in Spain: the PROTEUS study. Heroin Addiction and Related Clinical Problems, 13, 5-16.

Rose, V. J., Lutnick, A. y Kral, A. H. (2014). Feasibility of providing interventions for injection drug users in pharmacy settings: a case study among San Francisco pharmacists. Journal of Psychoactive Drugs, 46, 226-232. doi: 10.1080/02791072.2014.921745.

Torrens, M., Fonseca, F., Castillo, C. y Domingo-Salvany, A. (2013). Methadone maintenance treatment in Spain: the success of a harm reduction approach. Bulletin of the World Health Organization, 91, 136-141. 